\title{
Glu298Asp polymorphism influences the effects of beetroot bread on diastolic blood pressure in healthy men: A pilot study
}

\author{
D. A. Hobbs ${ }^{1,2}$, T. W. George ${ }^{3}$ and J. A. Lovegrove ${ }^{1,2}$ \\ ${ }^{1}$ Hugh Sinclair Unit of Human Nutrition, ${ }^{2}$ Institute for Cardiovascular and Metabolic Research (ICMR), University of \\ Reading, Whiteknights, PO Box 226, Reading, RG6 6AP and ${ }^{3}$ Department of Biology, Food and Nutritional Sciences, \\ Ellison Building, Ellison Place, Northumbria University, NE1 8ST, UK
}

Glu298Asp polymorphism of the endothelial nitric oxide synthase eNOS gene (NOS3) has previously been identified as a risk factor for hypertension $^{(1)}$ and coronary artery disease ${ }^{(2)}$. Studies suggest that the higher risk observed in thymine (T) allele carriers is due to endothelial dysfunction associated with lower eNOS activity ${ }^{(3)}$. It has been proposed that acute consumption of nitrate ameliorates postprandial endothelial dysfunction possibly by the donation of nitric oxide by a mechanism that does not rely on eNOS ${ }^{(4)}$. Nevertheless, how these factors interact has yet to be determined. Therefore, the aim of this study was to investigate whether the presence of NOS3 Glu298Asp polymorphism interacts with the nitrate content of beetroot-enriched bread to influence postprandial blood pressure.

An acute, randomised, single-blind, controlled, crossover pilot study was performed in fourteen healthy men (mean age 34 (SD 9) years and BMI 22.8 (SD 2) kg/m²) who were retrospectively genotyped for NOS3 Glu298asp polymorphism (7 GG; 7 GT/TT - T carriers). Volunteers were randomised to receive $200 \mathrm{~g}$ beetroot-enriched bread ( $1.1 \mathrm{mmol}$ nitrate) or control bread (no beetroot; $0.01 \mathrm{mmol}$ nitrate) on two separate occasions 10 days apart. Baseline and incremental area under the curve of blood pressure and nitrate/nitrite (NOx) were measured fasted and postprandially for a total period of 6 hours.

A treatment by genotype interaction was observed for diastolic blood pressure (DBP; $P<0.01$ ), in which the DBP was significantly lower in the $\mathrm{T}$ carriers $(P<0.01)$ only after beetroot compared with control bread. No significant treatment by genotype interaction was observed for SBP. However, there was a significant effect of treatment $(P<0.01)$ on plasma NOx regardless of genotype and a near significant treatment by genotype interaction $(P<0.06)$, with Bonferroni post-hoc tests revealing the $\mathrm{T}$ carriers had significantly higher plasma NOx $(P<0.01)$ after beetroot-enriched bread compared with control bread.
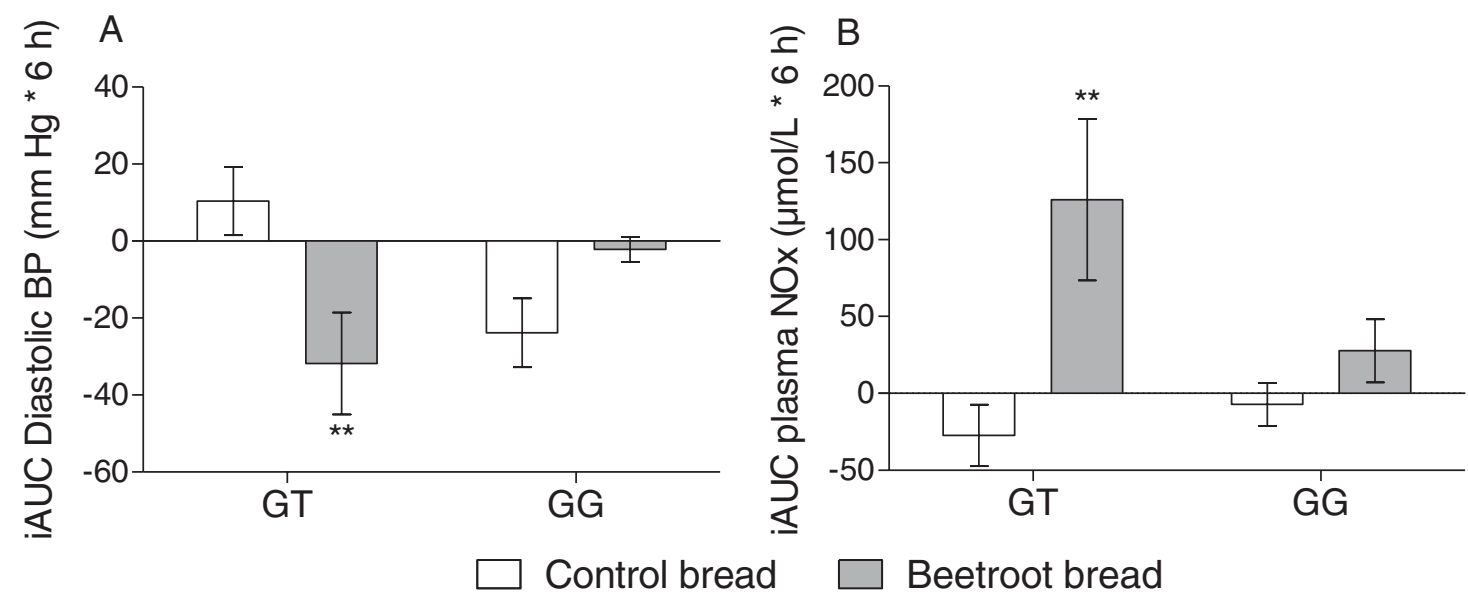

Diastolic BP (A) and plasma NOx $(B)$ responses in $G G(n=7)$ and T carriers $(n=7)$ of the NOS3 Glu298Asp polymorphism after acute ingestion of either control or beetroot-enriched bread. Data expressed as mean \pm SE. Significant treatment by genotype interaction for $D B P(* * P<0.01)$ and near significant treatment by genotype interaction for plasma NOx $(P<0.06)$ using 2 -way ANOVA, with the $T$ carriers showing significantly lower DBP and NOx responses (both **P<0.01) after beetroot-enriched vs. control bread after Bonferroni post-hoc test.

In conclusion, beneficial DBP reduction was observed only in the T carriers of the NOS3 Glu298Asp polymorphism after consumption of nitrate-rich beetroot bread. This data requires confirmation in a larger population group.

1. Hingorani AD et al. (1999) Circulation 100, 1515-20.

2. Miyamoto $\mathrm{Y}$ et al. (1998) Hypertension 32, 3-8.

3. Casas JP et al. (2006) Am J Epidemiol 164, 921-35.

4. Lundberg JO et al. (2006) Nitric Oxide 15, 359-62. 